PROCEEDINGS OF THE

AMERICAN MATHEMATICAL SOCIETY

Volume 140, Number 5, May 2012, Pages 1761-1767

S 0002-9939(2011)11046-3

Article electronically published on August 22, 2011

\title{
BOHR AND BESICOVITCH ALMOST PERIODIC DISCRETE SETS AND QUASICRYSTALS
}

\author{
S. FAVOROV
}

(Communicated by Mario Bonk)

\begin{abstract}
A discrete set $A$ in the Euclidean space is almost periodic if the measure with the unit masses at points of the set is almost periodic in the weak sense. We investigate properties of such sets in the case when $A-A$ is discrete. In particular, if $A$ is a Bohr almost periodic set, we prove that $A$ is a union of a finite number of translates of a certain full-rank lattice. If $A$ is a Besicovitch almost periodic set, then there exists a full-rank lattice such that in most cases a nonempty intersection of its translate with $A$ is large.
\end{abstract}

The notion of an almost periodic discrete set in the complex plane is well known in the theory of almost periodic holomorphic and meromorphic functions (cf. 13, [20], 7], 8], 4]). Almost periodic discrete sets in the $p$-dimensional Euclidean space appear later in the mathematical theory of quasicrystals. In particular, the various notions of almost periodicity are studied in [12] in connection with quasicrystals to capture the notion of a pure diffraction spectrum. Here the idea is that this should be associated to the spectrum of an almost periodic function. It is known that there is a large subclass of Meyer sets, called model sets (or cut and project sets), which have a pure point diffraction spectrum in a suitable sense, coming from ergodic theory. Some recent references are [16, [19, [2], and [17. These dynamical notions of pure point diffractivity appear different from Besicovitch almost periodicity, but with some similarities.

The paper 12 also discusses a notion of diffractivity given by Patterson sets; these were characterized in [9. Note that in [9], Theorem 1.1, the author used a kind of Besicovitch-like almost periodicity notion.

In this connection, the following question was raised in [12] (Problem 4.4): whether any Bohr almost periodic discrete set is a finite union of translations of a full-rank lattice in $\mathbb{R}^{p}$. In [5] and [6] we showed that every almost periodic perturbation of a full-rank lattice in $\mathbb{R}^{p}$ is a Bohr almost periodic set. Hence, there exists a wide class of such sets.

Next, most of the models of quasicrystals are sets of a finite type. In the present article we prove that every Bohr almost periodic discrete set of a finite type is just a finite union of translations of a full-rank lattice. We also prove that every Besicovitch almost periodic discrete set of a finite type has, in a sense, asymptotically the same form.

Received by the editors November 11, 2010 and, in revised form, January 11, 2011.

2010 Mathematics Subject Classification. Primary 52C23; Secondary 42A75, 52C07.

Key words and phrases. Quasicrystals, Bohr almost periodic set, Besicovitch almost periodic set, ideal crystal.

(C) 2011 American Mathematical Society
Reverts to public domain 28 years from publication 1761 
Let us recall some known definitions (see, for example, 1], [18]).

A continuous function $f(x)$ in $\mathbb{R}^{p}$ is almost periodic in the sense of Bohr if for any $\varepsilon>0$ the set of $\varepsilon$-almost periods of $f$

$$
E_{\varepsilon}=\left\{\tau \in \mathbb{R}^{p}: \sup _{x \in \mathbb{R}^{p}}|f(x+\tau)-f(x)|<\varepsilon\right\}
$$

is a relatively dense set in $\mathbb{R}^{p}$. The latter means that there is $R=R(\varepsilon)<\infty$ such that every ball of radius $R$ contains at least one $\varepsilon$-almost period of $f$.

The following definition is an evident generalization of the one given by Besicovitch in 3] for the case when $p=1$ : an integrable function $F(x)$ in $\mathbb{R}^{p}$ is almost periodic in the sense of Besicovitch, or $B^{1}$-almost periodic, if for any $\varepsilon>0$ there is a Bohr almost periodic function $f_{\varepsilon}(x)$ in $\mathbb{R}^{p}$ such that

$$
\limsup _{R \rightarrow \infty} \frac{1}{\omega_{p} R^{p}} \int_{|x|<R}\left|F(x)-f_{\varepsilon}(x)\right| d x<\varepsilon .
$$

Here $\omega_{p}$ is the volume of the unit ball in $\mathbb{R}^{p}$. Next, $T \in \mathbb{R}^{p}$ is an $\varepsilon$-almost period of $F$ in the sense of Besicovitch if

$$
\limsup _{R \rightarrow \infty} \frac{1}{\omega_{p} R^{p}} \int_{|x|<R}|F(x+T)-F(x)| d x<\varepsilon .
$$

It follows from the definition of $B^{1}$-almost periodicity that the set of $\varepsilon$-almost periods of $F$ in the sense of Besicovitch is relatively dense in $\mathbb{R}^{p}$ for each $\varepsilon>0$.

We will say that a set $A \subset \mathbb{R}^{p}$ is discrete if it has no finite limit points and is uniformly discrete if there is $r>0$ such that any ball of radius $r$ contains at most one point of $A$. A discrete set $A \subset \mathbb{R}^{p}$ is Bohr (Besicovitch) almost periodic if for every continuous function $\varphi$ in $\mathbb{R}^{p}$ with a compact support the sum $\sum_{a \in A} \varphi(x-a)$ is a Bohr (Besicovitch) almost periodic function. In other words, the union of the unit masses at the points of $A$ is a Bohr (Besicovitch) almost periodic measure in the weak sense.

There is a geometric criterion for a discrete set to be Bohr almost periodic.

Theorem 1 ([5], Theorem 11). A discrete set $A=\left(a_{n}\right)_{n \in \mathbb{N}} \subset \mathbb{R}^{p}$ is Bohr almost periodic if and only if for each $\varepsilon>0$ the set of $\varepsilon$-almost periods of $A$

$\left\{\tau \in \mathbb{R}^{p}: \exists\right.$ a bijection $\quad \sigma: \mathbb{N} \rightarrow \mathbb{N} \quad$ such that $\left.\quad\left|a_{n}+\tau-a_{\sigma(n)}\right|<\varepsilon \quad \forall n \in \mathbb{N}\right\}$ is relatively dense in $\mathbb{R}^{p}$.

It follows easily from this criterion that the number of elements of $A$ in every ball of radius 1 is uniformly bounded.

Note that for a uniformly discrete set $A$ and sufficiently small $\varepsilon>0$, a vector $\tau$ is an $\varepsilon$-almost period of $A$ if and only if for any $a \in A$ there exists $a^{\prime} \in A$ such that $\left|a+\tau-a^{\prime}\right|<\varepsilon$.

A set $A$ is a Delone set if it is uniformly discrete and relatively dense in $\mathbb{R}^{p}$. For sets $A, C \subset \mathbb{R}^{p}$ put $A+C=\{a+c: a \in A, c \in C\} ; A-C$ is defined in a similar way. Also, put $B(x, R)=\left\{y \in \mathbb{R}^{p}:|y-x|<R\right\}$. Following [11, we will say that a discrete set $A \subset \mathbb{R}^{p}$ is a set of finite type if the set $A-A$ is discrete. A set $A$ is $a$ Meyer set if the set $A-A$ is a Delone set 1 Clearly, any Meyer set is a Delone set.

\footnotetext{
${ }^{1}$ The notion of a Meyer set goes back to [14. There Meyer has a notion of a relatively dense harmonious set; the name Meyer set was assigned later by others (see [15]). Also, the original definition of a Meyer set is different from above. The equivalence to the definition used here was shown in 10].
} 
A set $A \subset \mathbb{R}^{p}$ is an ideal crystal if $A$ consists of a finite number of translates of a full-rank lattice $L$. That is, $A=L+F$, where $F$ is a finite set and $L$ is an additive discrete subgroup of $\mathbb{R}^{p}$ such that $\operatorname{Lin}_{\mathbb{R}} L=\mathbb{R}^{p}$.

We shall prove the following theorem.

Theorem 2. If a set A of a finite type is Bohr almost periodic, then it is an ideal crystal.

Proof. Every ball of radius $R=R(1)$ contains at least one 1-period of $A$; hence each ball of radius $R+1$ contains at least one point $a \in A$. Since the set $A-A$ is discrete, we see that there is $\varepsilon>0$ such that $\varepsilon<\min \{1 ;|(b-a)+(c-d)|\}$ whenever $a, b, c, d \in A$ and $|a-b|<2 R+4,|c-d|<2 R+4, a-b \neq c-d$. In particular, $\varepsilon<|a-b|$ whenever $a, b \in A$ and $a \neq b$.

Fix $a \in A$. Let $\tau \in \mathbb{R}^{p}$ be an arbitrary $(\varepsilon / 2)$-almost period of $A$. Taking into account our choice of $\varepsilon$, we see that there is a unique $c \in A$ that satisfies the inequality $|a+\tau-c|<\varepsilon / 2$. Clearly, $T=c-a$ is an $\varepsilon$-almost period of $A$. Let us show that $T$ is actually a period of $A$.

Suppose that $b \in A$ such that $b \neq a$ and $|a-b|<2 R+3$. Since $T$ is an $\varepsilon$-almost period of $A$, there exists a point $d \in A$ such that $|b+T-d|=|(a-b)-(c-d)|<\varepsilon$. Since $|c-d| \leq|a-b|+|b+T-d|<2 R+4$, we obtain $a-b=c-d$ and $d=b+T$. We repeat these arguments for all $b \in A$ such that $|b-a|<2 R+3$ and, after that, for all $b^{\prime} \in A$ such that $\left|b^{\prime}-b\right|<2 R+3$. After a countable number of steps we obtain that $a+T \in A$ for all $a \in A_{1} \subset A$. If $A \backslash A_{1} \neq \emptyset$, then $R_{1}=\inf \left\{|a-b|: a \in A_{1}, b \in A \backslash A_{1}\right\} \geq 2 R+3$. Take $a^{\prime} \in A_{1}$ and $b^{\prime} \in A \backslash A_{1}$ such that $\left|a^{\prime}-b^{\prime}\right|<R_{1}+1$. This implies that $B\left(\frac{a^{\prime}+b^{\prime}}{2}, R+1\right) \cap A_{1}=\emptyset$ and $B\left(\frac{a^{\prime}+b^{\prime}}{2}, R+1\right) \cap\left(A \backslash A_{1}\right)=\emptyset$, which is impossible. Hence, $A=A_{1}$ and $T$ is a period of $A$.

Next, take $(\varepsilon / 2)$-almost periods $\tau_{j}$ from the set

$$
\left\{x \in \mathbb{R}^{p}:|x|>4 p,\left|x-\left\langle x, e_{j}\right\rangle\right|<\frac{\left|\left\langle x, e_{j}\right\rangle\right|}{4 p}\right\}, \quad j=1, \ldots, p,
$$

where $e_{j}, j=1, \ldots, p$, is the intrinsic basis in $\mathbb{R}^{p}$. There are periods $T_{j}$ such that $\left|T_{j}-\tau_{j}\right|<1 / 2$. Since $\left|\left\langle\tau_{j}, e_{j}\right\rangle\right|>2 p$, we get

$$
\frac{\max _{k \neq j}\left|\left\langle T_{j}, e_{k}\right\rangle\right|}{\left|\left\langle T_{j}, e_{j}\right\rangle\right|} \leq \frac{\left|T_{j}-\left\langle T_{j}, e_{j}\right\rangle\right|}{\left|\left\langle T_{j}, e_{j}\right\rangle\right|}<\frac{\left|\tau_{j}-\left\langle\tau_{j}, e_{j}\right\rangle\right|+1}{\left|\left\langle\tau_{j}, e_{j}\right\rangle\right|-1 / 2}<\frac{1}{p}, \quad j=1, \ldots, p .
$$

Hence, the determinant of the matrix $\left(\left\langle T_{j}, e_{k}\right\rangle\right)_{j, k=1}^{p}$ does not vanish and the vectors $T_{1}, \ldots, T_{p}$ are linearly independent. Consequently, the set $L=\left\{n_{1} T_{1}+\cdots+n_{p} T_{p}\right.$ : $\left.n_{1}, \ldots, n_{p} \in \mathbb{Z}\right\}$ is a full-rank lattice. Next, the set $F=\left\{a \in A:|a|<\left|T_{1}\right|+\cdots+\right.$ $\left.\left|T_{p}\right|\right\}$ is finite. All vectors $t \in L$ are periods of $A$, hence, $L+F \subset A$. On the other hand, for each $a \in A$ there is $t \in L$ such that $|a-t|<\left|T_{1}\right|+\cdots+\left|T_{p}\right|$; hence, $a-t \in F$. The theorem is proved.

Besicovitch almost periodic Meyer sets form a strictly larger class than Bohr almost periodic Meyer sets. Such sets that are not ideal crystals can be obtained by perturbing the points in an ideal crystal in a bounded region.

Indeed, let $L$ be a full-rank lattice, $E$ be an arbitrary discrete set, and $r$ be an arbitrary positive number. Put $A=(L \backslash B(0, r)) \cup(E \cap B(0, r))$. It is easily seen that $A$ is a Meyer set. Also, in general, $A$ is not an ideal crystal. Next, for any continuous function $\varphi$ with a compact support the sum $\sum_{a \in L} \varphi(x-a)$ has 
$p$ linearly independent periods and coincides with $\sum_{a \in A} \varphi(x-a)$ for sufficiently large $x \in \mathbb{R}^{p}$. By definition, we obtain that the latter sum is a Besicovitch almost periodic function and $A$ is a Besicovitch almost periodic discrete set.

Nevertheless, we can prove a property of Besicovitch almost periodic Meyer sets that is close to the one proved in Theorem 2.

Let $A$ be a discrete set and let $L$ be a full-rank lattice. For $a, b \in A$ put $a \sim b$ if $a-b \in L$. Then there is a unique at most countable decomposition $A=\bigcup_{j} A_{j}^{L}$ into mutually disjoint equivalence classes $A_{j}^{L}$. Next, by $\# E$ denote the number of elements in the finite set $E$.

Theorem 3. Let a Besicovitch almost periodic discrete set $A \subset \mathbb{R}^{p}, p>1$, be a Meyer set. Then for any $N<\infty$ and $\eta>0$ there is a full-rank lattice $L$ such that

$$
\sum_{j: \#\left(A_{j}^{L} \cap B(0, R)\right)>N} \#\left(A_{j}^{L} \cap B(0, R)\right) \geq(1-\eta) \#(A \cap B(0, R)), \quad R>R(N, \eta) .
$$

Our proof is based on the following lemmas.

Lemma 1. Let a Delone set $A \subset \mathbb{R}^{p}$ be Besicovitch almost periodic. Then for any $\varepsilon>0$ and $\delta>0$ there is a relatively dense set $E=E(\varepsilon, \delta) \subset \mathbb{R}^{p}$ that satisfies for any $\tau \in E$

$$
\forall a \in A \backslash \tilde{A} \quad \exists a^{\prime} \in A: \quad\left|a+\tau-a^{\prime}\right|<\varepsilon,
$$

where $\tilde{A}=\tilde{A}(\tau) \subset A$ such that for sufficiently large $R$

$$
\#(\tilde{A} \cap B(0, R))<\delta \#(A \cap B(0, R)) .
$$

Proof. The set $A$ is relatively dense; therefore $A \cap B\left(x, R_{0}\right) \neq \emptyset$ for some $R_{0}<\infty$ and all $x \in \mathbb{R}^{p}$. Hence there exists $\kappa>0$ such that for all sufficiently large $R$

$$
\# A \cap B(0, R)>\kappa R^{p} \text {. }
$$

We may suppose that $\varepsilon<\frac{1}{2} \inf \{|a-b|: a, b \in A, a \neq b\}$. Let $\varphi$ be a $C^{\infty}$ function in $\mathbb{R}^{p}$ such that $0 \leq \varphi(x) \leq 1, \varphi(x)=1$ for $|x|<1 / 4$ and $\varphi(x)=0$ for $|x|>1 / 2$. Clearly, $\int \varphi(x) d x>\omega_{p} 4^{-p}$. The function $\psi(x)=\sum_{a \in A} \varphi\left(\frac{x-a}{\varepsilon}\right)$ is a Besicovitch almost periodic function. Clearly, if $\psi(x)>0$ for $x \in \mathbb{R}^{p}$, then $x \in B(a, \varepsilon / 2)$ for some $a \in A$. Let $E$ be the set of $\delta \kappa(\varepsilon / 4)^{p}$-almost periods of $\psi$ in the sense of Besicovitch and suppose $-\tau \in E$. Put $\tilde{A}=\{a \in A: B(a+\tau, \varepsilon) \cap A=\emptyset\}$. Since $\tilde{A} \subset A \backslash \operatorname{supp} \psi(x-\tau)$, we get that for $R>R(\tau)$

$$
\begin{gathered}
\omega_{p}(\varepsilon / 4)^{p} \#(\tilde{A} \cap B(0, R))<\sum_{a \in \tilde{A} \cap B(0, R)} \int_{B(a, \varepsilon / 2)} \varphi\left(\frac{x-a}{\varepsilon}\right) d x \\
\leq \int_{B(0, R+\varepsilon) \backslash \operatorname{supp} \psi(x-\tau)} \psi(x) d x \leq \int_{B(0, R+\varepsilon)}|\psi(x-\tau)-\psi(x)| d x<(\varepsilon / 4)^{p} \delta \kappa \omega_{p} R^{p} .
\end{gathered}
$$

Hence,

$$
\#(\tilde{A} \cap B(0, R))<\delta \kappa R^{p}
$$

The assertion of the lemma follows from (4).

Lemma 2. Suppose $E$ is a finite subset of a full-rank lattice $L=\operatorname{Lin}_{\mathbb{Z}}\left\{T_{1}, \ldots, T_{p}\right\} \subset$ $\mathbb{R}^{p}, p>1$. Put

$\operatorname{Fr}(E)=\left\{b \in E\right.$ : at least one of the points $b \pm T_{1}, \ldots, b \pm T_{p}$ does not belong to $\left.E\right\}$.

Then $(\# E)^{\frac{p-1}{p}} \leq(p-1) \# \operatorname{Fr}(E)$. 
Proof. Without loss of generality we may suppose $L=\mathbb{Z}^{p}$. For any $F \subset \mathbb{Z}^{p}$ denote by $P_{j}(F)$ the projection of $F$ on the hyperplane $x_{j}=0$. Note that $\# \operatorname{Fr}(E) \geq$ $\# P_{j}(E)$ for all $j \in \overline{1, p}$.

Put

$$
E_{1}=\left\{x \in E: \# E \cap P_{1}^{-I} P_{1}(\{x\}) \leq(\# E)^{1 / p}\right\},
$$

and, for each $j \in \overline{2, p-1}$ recurrently,

$$
E_{j}=\left\{x \in\left(E \backslash \bigcup_{i=1}^{j-1} E_{i}\right): \#\left(P_{j}^{-I} P_{j}(\{x\}) \cap\left(E \backslash \bigcup_{i=1}^{j-1} E_{i}\right)\right) \leq(\# E)^{1 / p}\right\} .
$$

Clearly, $\# E_{j} \leq \# P_{j}\left(E_{j}\right)(\# E)^{1 / p}$. If $\# E_{j} \geq(\# E) /(p-1)$ for some $j \in \overline{1, p-1}$, we get

$$
\# \operatorname{Fr}(E) \geq \# P_{j}(E) \geq \# P_{j}\left(E_{j}\right) \geq(p-1)^{-1}(\# E)^{1-1 / p} .
$$

If $\# E_{j}<(p-1)^{-1}(\# E)$ for all $j \in \overline{1, p-1}$, then there exists $x^{\prime} \in E \backslash \bigcup_{j=1}^{p-1} E_{j}$. Let $x_{p}^{\prime}$ be the last coordinate of $x^{\prime}$. Since $x^{\prime} \notin E_{p-1}$, we see that the set $P_{p-1}^{-I} P_{p-1}\left(\left\{x^{\prime}\right\}\right)$ $\cap\left(E \backslash \bigcup_{i=1}^{p-2} E_{i}\right)$ contains at least $(\# E)^{1 / p}$ points. Since every point $x^{\prime \prime}$ from the latter set does not belong to $E_{p-2}$, we get that all the sets $P_{p-2}^{-I} P_{p-2}\left(\left\{x^{\prime \prime}\right\}\right) \cap$ $\left(E \backslash \bigcup_{i=1}^{p-3} E_{i}\right)$ contain at least $(\# E)^{1 / p}$ points. Continuing this line of reasoning, we see that there exist at least $(\# E)^{\frac{p-1}{p}}$ different points in $E$ with the last coordinate $x_{p}^{\prime}$. Hence, these points have distinct projections on the hyperplane $x_{p}=0$. Consequently, in this case we get $\# \operatorname{Fr}(E) \geq \# P_{p}(E) \geq(\# E)^{\frac{p-1}{p}}$. The lemma follows.

Proof of the theorem. Note that $A$ is a Delone set. Applying Lemma 1 with $\varepsilon<$ $\frac{1}{2} \inf \{|x-y|: x, y \in A-A, x \neq y\}$ and $\delta=2^{-1} p^{-2} N^{-1 / p} \eta$, we find $\tau \in \mathbb{R}^{p}$ and $A(\tau) \subset A$ such that (2) and (3) hold. Replace $\tau$ by $T=a^{\prime}-a$. Taking into account the inequality $\left|a+\tau-a^{\prime}\right|<\varepsilon$ and the bound on $\varepsilon$, we get

$$
\forall a \in A \backslash \tilde{A}(\tau) \quad \exists a^{\prime} \in A: \quad a^{\prime}=a+T .
$$

Moreover, arguing as at the end of the proof of Theorem 2, we can take vectors $\tau_{1}, \ldots, \tau_{p}$ that are linearly independent over $\mathbb{R}$, vectors $T_{1}, \ldots, T_{p}$, and $A^{*}=$ $\bigcup_{j} \tilde{A}\left(\tau_{j}\right) \cup \bigcup_{j} \tilde{A}\left(-\tau_{j}\right)$ such that

$$
\forall a \in A \backslash A^{*} \quad \exists a_{j}^{\prime}, a_{j}^{\prime \prime} \in A: \quad a_{j}^{\prime}=a+T_{j}, \quad a_{j}^{\prime \prime}=a-T_{j}, \quad j \in \overline{1, p},
$$

where the set $A^{*}$ satisfies the following bound for all sufficiently large $R$ :

$$
\# A^{*} \cap B(0, R)<2 p \delta(\# A \cap B(0, R))=p^{-1} N^{-1 / p} \eta(\# A \cap B(0, R)) .
$$

Put $L=\left\{n_{1} T_{1}+\cdots+n_{p} T_{p}: n_{1}, \ldots, n_{p} \in \mathbb{Z}\right\}$ and $J=\left\{j: \#\left(A_{j}^{L} \cap B(0, R)\right) \leq N\right\}$. Taking into account Lemma 2 and the definition of $J$, we get

$$
\sum_{j \in J} \#\left(A_{j}^{L} \cap B(0, R)\right) \leq(p-1) \sum_{j \in J} \# F r\left(A_{j}^{L} \cap B(0, R)\right) N^{1 / p} .
$$

Every point $a \in \operatorname{Fr}\left(A_{j}^{L} \cap B(0, R)\right)$ such that $|a|+\max _{j}\left|T_{j}\right|<R$ belongs to $A^{*}$; therefore,

(7) $\sum_{j \in J} \# \operatorname{Fr}\left(A_{j}^{L} \cap B(0, R)\right) \leq \#\left[A^{*} \cap B(0, R)\right]+\# A \cap\left[B(0, R) \backslash B\left(0, R-\max _{j}\left|T_{j}\right|\right)\right]$. 
Since $A$ is a uniformly discrete and relatively dense set, we get

$$
\# A \cap\left[B(0, R) \backslash B\left(0, R-\max _{j}\left|T_{j}\right|\right)\right]=o(\# A \cap B(0, R)) \quad \text { as } \quad R \rightarrow \infty .
$$

It follows from (5)-(8) that

$$
\sum_{j \in J} \#\left(A_{j}^{L} \cap B(0, R)\right) \leq \eta \#(A \cap B(0, R)), \quad R>R(N, \eta) .
$$

The last inequality yields the assertion of the theorem.

We see that different notions of almost periodicity lead to different results. In particular, it looks like the almost periodicity in the sense of Bohr for discrete sets is too restrictive, and because of that it probably will have limited applications in mathematical theory of quasicrystals. It remains an interesting question to determine whether Besicovitch almost periodic Delone sets apply in classifying structures in the theory of quasicrystals.

\section{ACKNOWLEDGMENT}

The author thanks the referee for valuable recommendations and for pointing out a number of references on the subject.

\section{REFERENCES}

[1] J. Andres, A.M. Bersany, R.F. Grande, Hierarchy of almost-periodic function space, Rendiconti de Matematica (7) 26 (2006), 121-188. MR2275292 (2008b:43010)

[2] M. Baake, D. Lenz, R.V. Moody, Characterization of model sets by dynamical systems, Ergod. Th. Dyn. Systems 27 (2007), 341-382. MR2308136 (2008f:37007)

[3] A.S. Besicovitch, Almost periodic functions, Dover Publications, Inc., New York, 1955. Reprint by photo-offset of the 1st ed. [Cambridge, 1932]. MR0068029(16:817a)

[4] S. Favorov, Sunyer-i-Balaguer's almost elliptic functions and Yosida's normal functions, J. d'Analyse Math. 104 (2008), 307-340. MR2403439 (2009f:30066)

[5] S. Favorov, Ye. Kolbasina, Almost periodic discrete sets, Journal of Mathematical Physics, Analysis, Geometry 6 (2010), no. 1, 34-47, 135. MR2655763

[6] S. Favorov, Ye. Kolbasina, Perturbations of discrete lattices and almost periodic sets, Algebra and Discrete Math. 9 (2010), no. 2, 50-60. MR2808780

[7] S.Yu. Favorov, A.Yu. Rashkovskii, A.I. Ronkin, Almost periodic divisors in a strip, J. d'Analyse Math. 74 (1998), 325-345. MR1631678(99k:42016)

[8] S.Yu. Favorov, N.D. Parfyonova, Meromorphic almost periodic functions, Matematichni Studii 13, no. 2 (2000), 190-198. MR1776544(2001e:30045)

[9] J.-B. Gouéré. Quasicrystals and almost periodicity, Commun. Math. Phys. 255 (2005), 655-681. MR2135448 (2006j:52026)

[10] J.C. Lagarias, Meyer's concept of quasicrystals and quasiregular sets, Communications in Math. Physics 179 (1996), 365-376. MR1400744 (97g:52049)

[11] J.C. Lagarias, Geometric models for quasicrystals I. Delone set of finite type, Discr. and Comp. Geometry, 1998. MR:1668082 (99m:52029)

[12] J.C. Lagarias, Mathematical quasicrystals and the problem of diffraction, Directions in Mathematical Quasicrystals, M. Baake and R. Moody, eds., CRM Monograph series, Vol. 13, AMS, Providence RI, 2000, 61-93. MR1798989 (2001m:52032)

[13] B.Ja. Levin, Distributions of Zeros of Entire Functions. Transl. of Math. Monograph, Vol. 5, AMS, Providence, RI, 1980. MR.589888 (81k:30011)

[14] Y. Meyer, Algebraic Numbers and Harmonic Analysis. North-Holland, Amsterdam-London, 1972. MR0485769 (58:5579)

[15] R.V. Moody, Meyer's sets and their duals, R.V. Moody, ed., The Mathematics of Long-Range Order, NATO ASI Series C, Springer-Verlag, New York, 1997. MR 1460032 (98e:52029)

[16] R.V. Moody, N. Strungaru, Point sets and dynamic systems in the autocorrelation topology, Canad. Math. Bull 47 (2004), 82-99. MR2032271(2005e:52031) 
[17] E.A. Robinson, Jr., A Halmos-von Neumann theorem for model sets, and almost automorphic dynamical systems, in: Dynamics, ergodic theory and geometry, pp. 243-272, MSRI Publ. No. 54, Cambridge Univ. Press, Cambridge, 2007. MR2369449 (2010a:37019)

[18] L.I. Ronkin, Almost periodic distributions and divisors in tube domains, Zap. Nauchn. Sem. POMI 247 (1997), 210-236 (Russian). MR1692679 (2000m:46090)

[19] M. Schlottman, Generalized model sets and dynamic systems, in: Directions in Mathematical Quasicrystals, Amer. Math. Soc., Providence, RI, 2000, pp. 143-159. MR1798991 (2001k:52035)

[20] H. Tornehave, Systems of zeros of holomorphic almost periodic functions, Kobenhavns Universitet Matematisk Institut, preprint No. 30, 1988, 52 pp.

Mathematical School, Kharkov National University, Swobody sq. 4, Kharkov, 61077 UKRAINE

E-mail address: sfavorov@gmail.com 